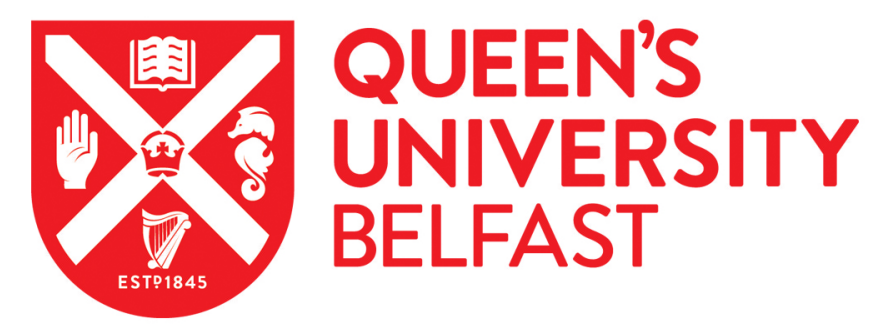

\title{
Multiple-probe electronic open boundaries with bad contacts
}

Todorov, T. N., \& Horsfield, A. P. (2019). Multiple-probe electronic open boundaries with bad contacts. Physical Review B, 99(4), [045415]. https://doi.org/10.1103/PhysRevB.99.045415

\author{
Published in: \\ Physical Review B
}

Document Version:

Publisher's PDF, also known as Version of record

Queen's University Belfast - Research Portal:

Link to publication record in Queen's University Belfast Research Portal

\section{Publisher rights}

(c) 2019 American Physical Society.

This work is made available online in accordance with the publisher's policies. Please refer to any applicable terms of use of the publisher.

\section{General rights}

Copyright for the publications made accessible via the Queen's University Belfast Research Portal is retained by the author(s) and / or other copyright owners and it is a condition of accessing these publications that users recognise and abide by the legal requirements associated with these rights.

Take down policy

The Research Portal is Queen's institutional repository that provides access to Queen's research output. Every effort has been made to ensure that content in the Research Portal does not infringe any person's rights, or applicable UK laws. If you discover content in the Research Portal that you believe breaches copyright or violates any law, please contact openaccess@qub.ac.uk. 


\title{
Multiple-probe electronic open boundaries with bad contacts
}

\author{
Tchavdar N. Todorov* \\ Atomistic Simulation Centre, School of Mathematics and Physics, Queen's University Belfast, Belfast BT7 1NN, United Kingdom \\ Andrew P. Horsfield ${ }^{\dagger}$ \\ Department of Materials and Thomas Young Centre, Imperial College London, London SW7 2AZ, United Kingdom
}

(Received 16 October 2018; revised manuscript received 13 December 2018; published 10 January 2019)

\begin{abstract}
We revisit the weak-coupling limit of the hairy probes method for electronic open boundaries. In this limit, the electronic density matrix is approximately stationary. We exploit this fact to combine hairy probes with electron-phonon scattering at the level of low-order transitions between eigenstates of the electronic Hamiltonian. This provides a method that is computationally very efficient, and whose results can be interpreted quite straightforwardly. The resultant time-dependent hybrid method is illustrated through the numerical calibration of a thermoelectric nanoscale thermometer.
\end{abstract}

DOI: 10.1103/PhysRevB.99.045415

\section{INTRODUCTION}

Electrical contacts in real life can be good or bad. This is equally true of macroscopic circuitry and molecular-scale electronics. There are important situations where low electronic transmission (bad contacts) is actually desirable: a notable case is an electrochemical cell (for example, a battery) where electrons need to be able to enter and leave from outside, but the current internally is carried by the drift and diffusion of ions [1,2]. An interesting question is, therefore, are there benefits to be derived theoretically from deliberately considering bad contacts?

In recent work $[1,2]$ we have shown that a method for electronic open boundaries called hairy probes (HP) [3] affords key simplifications in the limit of weak HP-system coupling. The HP method considers a possibly long but finite leadsample-lead system, embedded in a sea of external probes, the HP. The defining feature of the method is that the HP, but not the system itself, are in the wide-band limit. As a result, the embedding self-energy for the system is energy independent and time local. This makes evaluation of the single-particle density matrix (DM) describing the state of the system very efficient.

In the weak-coupling limit, the HP connect only weakly to points in the system. As a result, the electronic DM becomes approximately diagonal in the eigenstates of the electronic Hamiltonian.

In the present paper we exploit this fact, to combine HP in the weak-coupling limit with a system of electron-phonon kinetic equations, based on low-order perturbation theory (Fermi's golden rule), which describe electron-phonon transitions in the system [4,5]. Like the HP open-boundary terms in the weak-coupling limit, these rate equations work with the populations of the eigenstates of the Hamiltonian. The

\footnotetext{
*t.todorov@qub.ac.uk

†a.horsfield@imperial.ac.uk
}

resultant hybrid method is formulated in the time domain, and represents the simplest approach to time-dependent inelastic transport that can be achieved with HP. We illustrate the success of this approach through the calibration, by real-time quantum-mechanical simulations, of a nanoscale thermometer, based on thermoelectricity $[5,6]$.

\section{METHOD}

The generic assembly we consider in the HP method is shown schematically in Fig. 1. We have a possibly long but finite system that can (but does not have to) be divided into two electrodes and a central region. We note that HP is by construction a multiterminal method. We allow the system to sustain atomic vibrations. Here we consider single-orbital orthogonal tight-binding models. More general descriptions of the electronic structure have been discussed in our past work [1].

Each of the two electrodes has a region where every atomic site, $n$, is connected to an external probe. These are the HP. The probe connected to site $n$ has its own electrochemical potential and temperature, with a corresponding electronic distribution function $f_{n}(E)$ (where $E$ is electron energy), and couples to the site with a strength $\Gamma_{n}$. (A site without a probe may be regarded as a site with a probe with $\Gamma_{n}=0$.) As already mentioned, the HP are assumed to lie in the wide-band limit; this is a significant part of the utility of the scheme.

\section{A. HP open boundaries}

The equation of motion for a DM is the quantum Liouville equation. If only a part of the matrix is of interest, then the equation of motion has two parts: the usual quantum Liouville expression for the part under consideration, plus an additional term describing the interaction of the implicit environment. HP augment the equation of motion for the one-electron DM, 


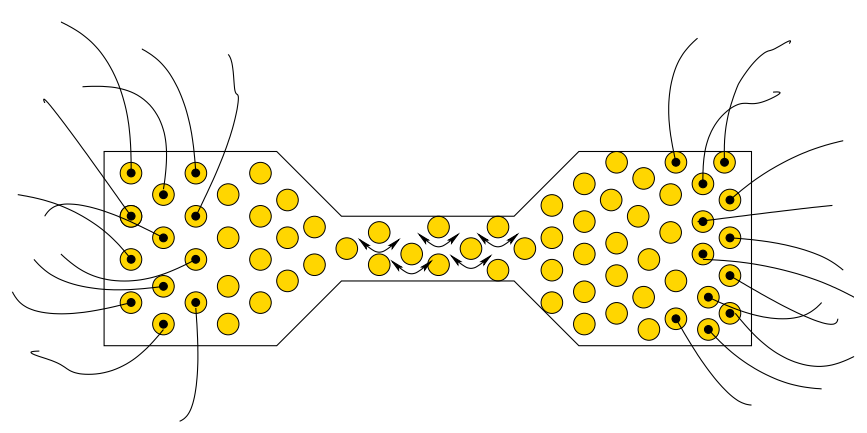

FIG. 1. Generic assembly in the hairy probes approach. See text for details.

$\rho$, through the terms [3]

$$
\begin{aligned}
\dot{\rho}^{\mathrm{HP}}= & \frac{1}{\mathrm{i} \hbar}\left(\Sigma^{+} \rho-\text { H.c. }\right) \\
& +\frac{1}{\mathrm{i} \hbar} \int_{-\infty}^{\infty}\left[\Sigma^{<}(E) G^{-}(E)-\text { H.c. }\right] d E,
\end{aligned}
$$

where

$$
\begin{gathered}
\Sigma^{ \pm}=\sum_{n} \Sigma_{n}^{ \pm}, \quad \Sigma_{n}^{ \pm}=\mp \mathrm{i} \frac{\Gamma_{n}}{2} P_{n} \\
G^{ \pm}(E)=\left(E-H-\Sigma^{ \pm} \pm \mathrm{i} \epsilon\right)^{-1} \\
\Sigma^{<}(E)=\sum_{n} \Sigma_{n}^{<}(E), \quad \Sigma_{n}^{<}(E)=\frac{1}{2 \pi} \Gamma_{n} f_{n}(E) P_{n}
\end{gathered}
$$

with $P_{n}=|n\rangle\langle n|$ the projection operator on real-space basis state $|n\rangle$ in our system. $H$ is the one-electron Hamiltonian, which for present purposes we assume to be non-self-consistent. The introduction of mean-field electronelectron screening, or possibly more sophisticated models of electron-electron interactions, is left as an extension.

The above equations result from projecting the equation of motion for the global DM onto the (explicit) system, composed of the (finite) electrodes and the central region. The main approximation is twofold. First, we impose the wide-band limit in the HP. This is beneficial, as indicated earlier. As discussed in Ref. [3], furthermore, it enables a controlled connection with the usual two-terminal Landauer solution, by taking the (double) limit of long leads (first) and small lead-HP coupling (second). It is important that imposing the wide-band limit in the probes is a weaker approximation than doing so directly in the electrodes: this would wash out their electronic structure, retained though possibly broadened in our case. Second, in the presence of dynamics in the central region we have to, formally, suppress multiple scattering and interference with the HP. This is the job of the parameter $\epsilon$ in Eq. (3). Alternatively, we have to make the distance between the HP and the dynamical region as large as possible. The quantity $\epsilon$ could, further, model a generic dephasing mechanism. In the above equations, $\Gamma_{n}$ enters in two ways: explicitly, via $\Sigma$, and implicitly, via $G$. The first causes direct communication between the system and HP. The second introduces a finite electron lifetime in the regions to which HP are coupled.
We now impose weak HP-system coupling by working to lowest order in $\Gamma_{n}$, whereupon Eq. (3) becomes $G^{ \pm}(E)=$ $(E-H \pm \mathrm{i} \epsilon)^{-1}$. The condition for this limit is that the electron mean-free path due to the HP [1] should be larger than the typical linear dimension of the regions with HP. It is known [2] that the resultant steady-state DM is close to diagonal in the eigenbasis of $H$. We thus adopt this basis as a natural representation of electronic states, and assume that the DM remains close to stationary at all times. The contribution of HP to the rate of change of the occupancy, $f_{\alpha}$, of eigenstate $|\alpha\rangle$ of $H$, with eigenvalue $E_{\alpha}$, becomes

$$
\begin{aligned}
\dot{f}_{\alpha}^{\mathrm{HP}} & =\left\langle\alpha\left|\dot{\rho}^{\mathrm{HP}}\right| \alpha\right\rangle \\
& =-\sum_{n} \frac{\Gamma_{n}}{\hbar}\left\langle\alpha\left|P_{n}\right| \alpha\right\rangle\left[f_{\alpha}-f_{n}\left(E_{\alpha} ; \epsilon\right)\right],
\end{aligned}
$$

where

$$
f_{n}\left(E_{\alpha} ; \epsilon\right)=\int_{-\infty}^{\infty} f_{n}(E) \frac{\epsilon / \pi}{\left(E-E_{\alpha}\right)^{2}+\epsilon^{2}} d E .
$$

We simplify the situation by working in the limit

$$
\lim _{\epsilon \rightarrow 0^{+}} f_{n}\left(E_{\alpha} ; \epsilon\right)=f_{n}\left(E_{\alpha}\right) .
$$

Then Eq. (6) is readily recognized as Fermi's golden rule for HP-system excursions.

We can furthermore obtain the electron particle current in probe $n$ as

$$
\begin{aligned}
J_{n}= & \operatorname{Tr}\left\{\frac{1}{\mathrm{i} \hbar}\left(\Sigma_{n}^{+} \rho-\text { H.c. }\right)\right. \\
& \left.+\frac{1}{\mathrm{i} \hbar} \int_{-\infty}^{\infty}\left[\Sigma_{n}^{<}(E) G^{-}(E)-\text { H.c. }\right] d E\right\} \\
= & -\sum_{\alpha} \frac{\Gamma_{n}}{\hbar}\left\langle\alpha\left|P_{n}\right| \alpha\right\rangle\left[f_{\alpha}-f_{n}\left(E_{\alpha}\right)\right],
\end{aligned}
$$

which can be recognized as Fermi's golden rule for excursions between the system and that probe. $J_{n}>0$ corresponds to net flow from probe $n$ into the system. When giving numerical values and in plots later, we will report particle current times $|e|$.

\section{B. Electron-phonon interactions}

We now allow the electrons to interact with a set of quantum harmonic oscillators, representing vibrational modes within our system, via the Hamiltonian

$$
\begin{aligned}
\mathcal{H}= & \sum_{\alpha} E_{\alpha} c_{\alpha}^{\dagger} c_{\alpha}+\sum_{j} \hbar \omega_{j}\left(a_{j}^{\dagger} a_{j}+1 / 2\right) \\
& -\sqrt{\frac{\hbar}{2 M \omega_{j}}} \sum_{\alpha, \beta, j} F_{\alpha \beta j} c_{\alpha}^{\dagger} c_{\beta}\left(a_{j}^{\dagger}+a_{j}\right) .
\end{aligned}
$$

Here $\left\{c_{\alpha}\right\}$ are a set of fermion annihilation operators corresponding to the eigenstates of the one-electron Hamiltonian $H$ introduced earlier, $\left\{a_{j}\right\}$ are boson annihilation operators for the set of quantum oscillators with angular frequencies $\left\{\omega_{j}\right\}$, and $M$ is the oscillator mass (which we assume to be the same for all of them but which can be made oscillator 
specific). The matrix elements $F_{\alpha \beta j}$ can be obtained from a chosen explicit electron-phonon coupling. In our tight-binding implementation

$$
F_{\alpha \beta j}=-\left\langle\alpha\left|\frac{\partial H}{\partial X_{j}}\right| \beta\right\rangle,
$$

where $X_{j}$ is the generalized displacement for vibrational mode $j$.

We again assume that the electron-phonon system remains close to an instantaneous stationary state, with occupancies allowed to evolve according to lowest-order perturbation theory. Then the rate of change of the occupancy of electronic state $|\alpha\rangle$ is given by [7]

$$
\begin{aligned}
\dot{f}_{\alpha}^{\text {eph }}= & \frac{2 \pi}{\hbar} \sum_{\beta, j} \frac{\hbar}{2 M \omega_{j}}\left|F_{\alpha \beta j}\right|^{2}\left[-f_{\alpha}\left(1-f_{\beta}\right) N_{j}\right. \\
& \left.+f_{\beta}\left(1-f_{\alpha}\right)\left(N_{j}+1\right)\right] \delta\left(E_{\alpha}-E_{\beta}+\hbar \omega_{j}\right) \\
& +\frac{2 \pi}{\hbar} \sum_{\beta, j} \frac{\hbar}{2 M \omega_{j}}\left|F_{\alpha \beta j}\right|^{2}\left[f_{\beta}\left(1-f_{\alpha}\right) N_{j}\right. \\
& \left.-f_{\alpha}\left(1-f_{\beta}\right)\left(N_{j}+1\right)\right] \delta\left(E_{\alpha}-E_{\beta}-\hbar \omega_{j}\right) .
\end{aligned}
$$

The rate of change of the occupancy of vibrational mode $j$ is given by

$$
\begin{aligned}
\dot{N}_{j}= & \frac{2 \pi}{\hbar} \sum_{\alpha, \beta} \frac{\hbar}{2 M \omega_{j}}\left|F_{\alpha \beta j}\right|^{2}\left[-f_{\alpha}\left(1-f_{\beta}\right) N_{j}\right. \\
& \left.+f_{\beta}\left(1-f_{\alpha}\right)\left(N_{j}+1\right)\right] \delta\left(E_{\alpha}-E_{\beta}+\hbar \omega_{j}\right) .
\end{aligned}
$$

Equation (13) conserves particle number, $\sum_{\alpha} \dot{f}_{\alpha}=0$, while Eqs. (13) and (14) conserve the total energy $E=\sum_{\alpha} f_{\alpha} E_{\alpha}+\sum_{j}\left(N_{j}+1 / 2\right) \hbar \omega_{j}$. These equations further obey detailed balance, meaning that if we plug in the Fermi-Dirac and Bose-Einstein distributions, namely $f_{\alpha}=\left[\mathrm{e}^{\left(E_{\alpha}-\mu\right) / k T_{e}}+1\right]^{-1}$ and $N_{j}=\left(\mathrm{e}^{\hbar \omega_{j} / k T_{\mathrm{ph}}}-1\right)^{-1}$, then all transition rates vanish, $\dot{f}_{\alpha}=0, \dot{N}_{j}=0$, when $T_{e}=T_{\mathrm{ph}}$. Therefore the equations are suitable for describing thermodynamically correct energy exchange and thermalization.

In practical implementations for finite systems with discrete levels, it is necessary to replace the energy-conserving $\delta$ functions above by a chosen normalized narrowly peaked function of finite width (Gaussians, in our test calculations later on). Electron particle number remains conserved identically, but the other properties above can then be violated. A way to enforce total energy conservation is to replace Eq. (14) by

$$
\begin{aligned}
\dot{N}_{j}= & \frac{2 \pi}{\hbar} \sum_{\alpha, \beta} \frac{\hbar}{2 M \omega_{j}}\left|F_{\alpha \beta j}\right|^{2}\left[-f_{\alpha}\left(1-f_{\beta}\right) N_{j}\right. \\
& \left.+f_{\beta}\left(1-f_{\alpha}\right)\left(N_{j}+1\right)\right] \\
& \times \frac{\left(E_{\beta}-E_{\alpha}\right)}{\hbar \omega_{j}} \delta\left(E_{\alpha}-E_{\beta}+\hbar \omega_{j}\right) .
\end{aligned}
$$

The main physical limitations of the kinetic model above are the quasistationary assumption (i.e., that the DM always commutes with the unperturbed Hamiltonian, the sole evolution being in the occupation numbers) and the suppression of coherence. Thus, it is unsuitable for fast coherent dynamics or highly nonstationary situations. We have furthermore treated charge injection and extraction (via the HP) and electron-phonon scattering as independent processes, neglecting higher-order interplay between the two. The strengths of the kinetic model are the flexibility in the choice of unperturbed electronic Hamiltonian, vibrational modes and the coupling between the two, and the computational tractability [4].

\section{Special cases}

As a reality check, we now find the steady-state occupations, in the absence of phonons, by setting the left-hand side of Eq. (6) equal to zero and solving for $f_{\alpha}$. This gives

$$
f_{\alpha}=\frac{\sum_{n} \Gamma_{n}\left\langle\alpha\left|P_{n}\right| \alpha\right\rangle f_{n}\left(E_{\alpha}\right)}{\sum_{n} \Gamma_{n}\left\langle\alpha\left|P_{n}\right| \alpha\right\rangle} .
$$

This agrees with Ref. [2], and corresponds to the population of an eigenstate being a weighted average of the populations of all the HP at the energy of the state.

Next, we imagine that $f_{\alpha}$ are maintained at a reference distribution $f_{\alpha}=f\left(E_{\alpha}\right)$. This could (but need not) be the equilibrium Fermi-Dirac distribution. We now ask for a condition on the probe distribution $f_{n}(E)$ such as to make probe $n$ carry zero net current and thus be in equilibrium with the system. It is evident from Eq. (10) that a sufficient condition for $J_{n}=0$ is $f_{n}(E)=f(E)$, i.e., that the probe distribution is the same as that maintained in the system.

We now examine our model, including phonons, under global equilibrium. By that we mean that all HP are maintained at a common electrochemical potential and electron temperature, and the phonons are maintained at that same temperature. The only remaining variables, therefore, are the occupations of electronic states in the system. We will show that setting $f_{\alpha}=f_{\mathrm{FD}}\left(E_{\alpha}\right)$, where $f_{\mathrm{FD}}$ is the Fermi-Dirac distribution for the given HP electrochemical potential and temperature, produces a stationary solution for the entire system. First, with the above assumption about the phonon temperature, each line in Eq. (13) vanishes individually. Similarly, Eq. (14) vanishes, as noted earlier, and the phonons are thus at equilibrium. Next, the expression in square brackets in Eq. (6) vanishes by construction. This means that the total rate of change of $f_{\alpha}$ vanishes. Thus the electronic subsystem is also at equilibrium. Finally, owing to the vanishing of the expression in square brackets, Eq. (10) vanishes. Thus there are no net currents in the HP. In these respects, we have a global state of equilibrium of the constituents: the HP, and the electronic and phonon subsystems of our system.

We conclude with a property akin to the zeroth law of thermodynamics for the phonon-free case. We have allowed each level in the system to equilibrate with the probes according to Eq. (16). Consider now the current in a particular probe, $n$. From Eq. (10), after substituting in for $f_{\alpha}$, we obtain

$$
-\hbar J_{n}=\sum_{\alpha} \Gamma_{n} P_{n \alpha} \frac{\sum_{m} \Gamma_{m} P_{m \alpha}\left[f_{m}\left(E_{\alpha}\right)-f_{n}\left(E_{\alpha}\right)\right]}{\sum_{m} \Gamma_{m} P_{m \alpha}},
$$

where $P_{n \alpha}=\left\langle\alpha\left|P_{n}\right| \alpha\right\rangle$. We therefore see that if the probe distribution functions are all the same, $f_{n}(E)=f_{m}(E), \forall m, n$, 
then all probe currents vanish, $J_{n}=0, \forall n$. Thus, whatever the couplings and locations of HP, if the HP start off with the same occupation functions, they will remain at equilibrium after we have allowed them to communicate via the system.

\section{THERMOMETER FUNCTIONALITY}

The rate equations describe the dynamics of a system of coupled electrons and phonons. We can use this to determine how an electron current is modified by the temperature of the phonons, and hence construct a thermometer that reports on the temperature of the phonons through a measurable electric current. The simplest system that displays thermometer functionality, and that can be studied with our method, is a two-site system, with the two resultant molecular orbitals coupled by a single phonon mode [8]. Each site is connected to its own HP. We should then expect that if there is a spatial asymmetry in the system, and there is a temperature difference between the electrons and the oscillator, a net electron current can flow even at zero applied bias [9]. (If there is left-right symmetry in the system, then the lead-to-lead current will have to vanish, by virtue of the symmetry.)

We label the two sites as $L$ and $R$, and the two molecular orbitals as $\alpha$ and $\beta$, with $E_{\beta} \sim E_{\alpha}-\hbar \omega$, where $\omega$ is the angular frequency of the oscillator. A key ingredient in the model is that the molecular orbitals are spatially asymmetric, with

$$
P_{L \alpha} \neq P_{R \alpha}, \quad P_{L \beta} \neq P_{R \beta},
$$

where the notation was defined after Eq. (17).

There are two HP, one per site. They have identical distribution functions, $f_{n}(E)=f_{\mathrm{FD}}(E), n=L, R$. We take $\Gamma_{L}=$ $\Gamma_{R}=\Gamma$. The phonon mode, coupling the molecular states $\alpha$ and $\beta$, is maintained at a different temperature from that of the HP.

We introduce the quantity

$$
\begin{aligned}
Q= & \frac{2 \pi}{\hbar} \frac{\hbar}{2 M \omega}\left|F_{\alpha \beta}\right|^{2}\left[f_{\beta}\left(1-f_{\alpha}\right) N-f_{\alpha}\left(1-f_{\beta}\right)(N+1)\right] \\
& \times \delta\left(E_{\alpha}-E_{\beta}-\hbar \omega\right),
\end{aligned}
$$

which gives two physical quantities, as can be seen from Eqs. (13) and (14). First, it gives the phonon-induced transition rate from electronic state $\beta$ into electronic state $\alpha$ on the dimer. Second, to within a factor of $\hbar \omega$, it gives the energy current out of the notional thermostat that keeps the phonon mode at a fixed temperature.

Because the HP temperature and the phonon temperature are different, we allow $Q$ to be nonzero in the steady state. We now move on to constructing this steady state. From Eqs. (13) and (6) we get:

$$
\begin{aligned}
-\frac{\Gamma}{\hbar}\left[f_{\alpha}-f_{\mathrm{FD}}\left(E_{\alpha}\right)\right]+Q & =0 \\
-\frac{\Gamma}{\hbar}\left[f_{\beta}-f_{\mathrm{FD}}\left(E_{\beta}\right)\right]-Q & =0 .
\end{aligned}
$$

Next we consider the currents in the two HP. From Eq. (10), using Eqs. (20) and (21), we get

$$
J_{L}+J_{R}=-\left(P_{L \alpha}+P_{R \alpha}\right) Q+\left(P_{L \beta}+P_{R \beta}\right) Q .
$$

But $P_{L \alpha}+P_{R \alpha}=1=P_{L \beta}+P_{R \beta}$ and thus $J_{L}+J_{R}=0$. This establishes continuity of current. We also find

$J=\left(J_{L}-J_{R}\right) / 2=\left[-\left(P_{L \alpha}-P_{R \alpha}\right) Q+\left(P_{L \beta}-P_{R \beta}\right) Q\right] / 2$.

This is the average current at the $L$ and $R$ probe-system interfaces. We see that, by virtue of Eq. (18) (the breaking of left-right symmetry), if $Q \neq 0$, then $J \neq 0$. Thus the system responds with electric current to a temperature difference between vibrations in the system and the electron baths (the HP environment).

\section{THERMOMETER CALIBRATION}

Finally, we show how our method can be used to calibrate a thermometer based on the above principle. We consider a bimetallic junction, which we model as a linear atomic chain, of length 200 atoms, with the two halves of the chain having different on-site energies. We take the nearest-neighbor hopping integral to be $A=-1 \mathrm{eV}$ (producing a bandwidth of $4 \mathrm{eV}$ ). The on-site energy in the left half of the chain is $-1 \mathrm{eV}$ and in the right half it is $+1 \mathrm{eV}$. We will consider situations in which all HP have the same electrochemical potential, $\mu=0$.

$\mathrm{HP}$ are applied to the first five and the last five atoms in the chain. For those $\mathrm{HP} \Gamma_{n}=\Gamma=0.1 \mathrm{eV}$. The regions with HP are characterized by an electronic mean-free path $\lambda \sim 2|A| / \Gamma$ atomic sites [1]. In our case $\lambda \sim 20$, meeting the weak-coupling requirement that $\lambda$ should exceed the linear dimension of the HP regions.

The middle ten atoms are treated as Einstein oscillators with equal frequencies, $\hbar \omega=0.2 \mathrm{eV}$ and $M=1 \mathrm{amu}$. The coupling operator for an oscillator at site $j$ is

$F_{j}=f_{j}(|j\rangle\langle j-1|+| j-1\rangle\langle j|-| j\rangle\langle j+1|-| j+1\rangle\langle j|)$,

which corresponds to the variation of electronic hopping rate between neighboring sites with atomic displacement. In our case all $f_{j}=-2 \mathrm{eV} / \AA$.

The energy-conserving $\delta$ functions in electron-phonon transition rates are represented by Gaussians of standard deviation $\Delta E=0.03 \mathrm{eV}$. Each half of the chain has a mean electronic level spacing $\delta E \sim$ bandwidth/length $\sim 0.04 \mathrm{eV}$. Thus, our choice of broadening amounts to $\Delta E \sim \delta E$. The justification for this choice of broadening is that Fermi's golden rule envisages a continuum of final states, which we do not have; instead we have a dense set of discrete levels; to recover golden rule results, we thus require $\delta E$ to be less than, or at most similar to, $\Delta E$. One possible physical origin of the broadening are the HP themselves, which provide a continuum of electronic states. However we have assumed very small coupling; we are compensating for this here.

We propagate the occupations of electronic states according to the equation of motion

$$
\dot{f}_{\alpha}=\dot{f}_{\alpha}^{\mathrm{HP}}+\dot{f}_{\alpha}^{\mathrm{eph}} .
$$

In the present application, the phonon occupancies are kept fixed in time, corresponding to the phonons being coupled very efficiently to an external bath. Let now $J_{L / R}$ denote the sum of currents in the HP at the left/right end of the system. 


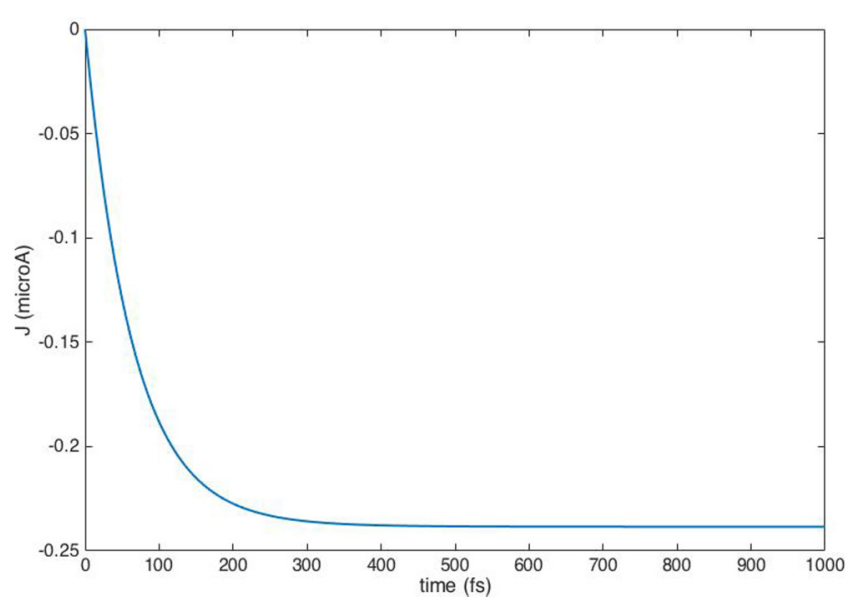

FIG. 2. Current $J$ as a function of time for the parameters in the text.

We calculate the average current $J=\left(J_{L}-J_{R}\right) / 2$ (including spin degeneracy).

Figure 2 shows $J$ as a function of time for a probe temperature $k T_{e}=0.1 \mathrm{eV}$ and phonon temperature $k T_{\mathrm{ph}}=0.15 \mathrm{eV}$, starting from electronic occupations, $f_{\alpha}$, of the system states, given by the Fermi-Dirac distribution for the given $T_{e}$. We see that the current is nonzero and settles at an eventual steady-state value $J_{\infty}$, which in practice we take to be the current at the end of the run of the above duration.

We now calculate this steady-state current for different $T_{\mathrm{ph}}$ and a given $T_{e}$, characterized by the fractional temperature difference $\delta T=\left(T_{\mathrm{ph}}-T_{e}\right) / T_{e}$. There is one subtlety in the procedure. Due to the finite broadening of the energy $\delta$ functions above, $J_{\infty}$ at $\delta T=0$ is not exactly zero. We thus calculate the difference $J_{\infty}(\delta T)-J_{\infty}(0)$, for every $\delta T$ considered, and term this difference net current.

Figure 3 shows the net current as a function of $\delta T$ for $k T_{e}=0.1 \mathrm{eV}$.

We see that the currents are appreciable. Thus we have a system that measures an applied temperature difference between vibrations and the external probes by generating measurable current flow at zero bias.

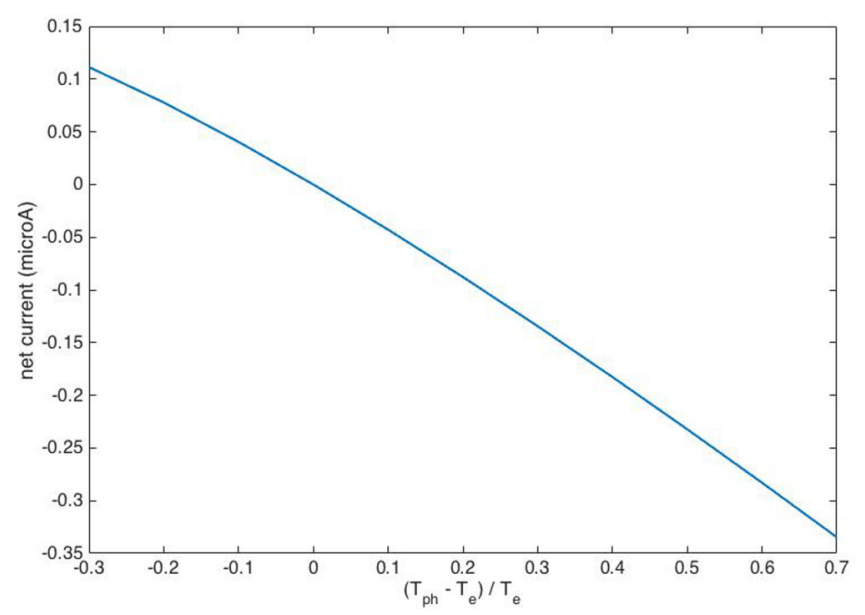

FIG. 3. Net current $J_{\infty}(\delta T)-J_{\infty}(0)$ as a function of fractional temperature difference $\delta T$ (see text) for $k T_{e}=0.1 \mathrm{eV}$.

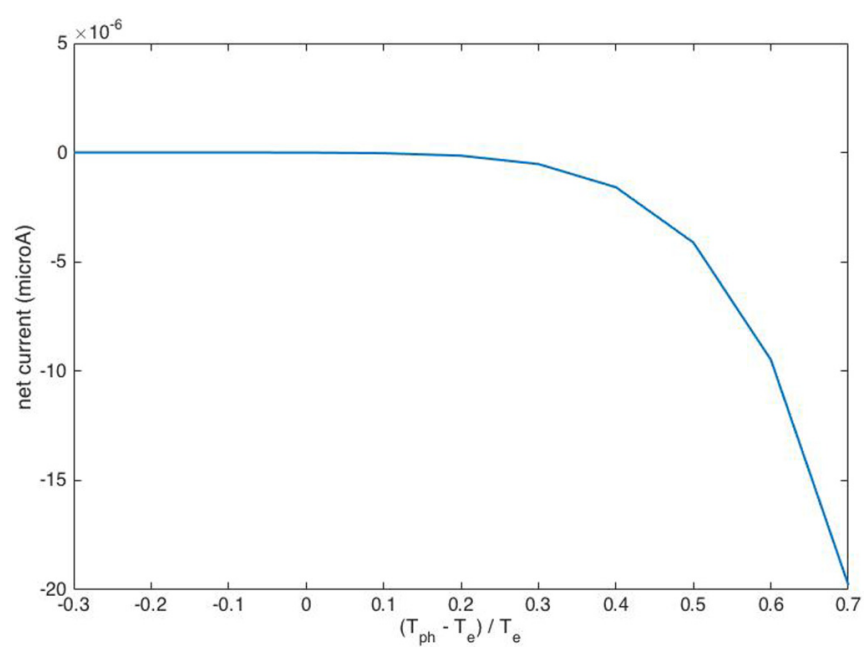

FIG. 4. Net current $J_{\infty}(\delta T)-J_{\infty}(0)$ as a function of fractional temperature difference $\delta T$ (see text) for $k T_{e}=0.01 \mathrm{eV}$.

The situation is dramatically different at low temperature. Figure 4 shows the net current as a function of $\delta T$ for $k T_{e}=0.01 \mathrm{eV}$. We see that the currents are tiny. The reason is that at low temperature, with all HP at the same electrochemical potential, phonon emission by electrons is mostly blocked by Pauli exclusion. Thus phonon-assisted electron transfer must of necessity involve phonon absorption. But that is also mostly frozen out at low temperature, as the typical phonon occupancy $N \sim \mathrm{e}^{-\hbar \omega / k T_{\mathrm{ph}}}$. Hence the low transition rates and low currents. Indeed, the currents pick up dramatically with increasing $T_{\mathrm{ph}}$ (and thus with increasing $\delta T$ ). In fact, the resultant behavior furnishes the basis for a rectifier, which responds dramatically to temperature changes of one sign and remains inactive otherwise.

We note that, for the above reasons, the low-temperature thermometer becomes more promising with decreasing $\hbar \omega$. Sensitivity is gained both due to the gain in phonon occupancy and due to the factors of $1 / \omega$ in transition rates. For example, reducing $\hbar \omega$ from $0.2 \mathrm{eV}$ to $0.1 \mathrm{eV}$ pushes up the net current for $k T_{e}=0.01 \mathrm{eV}$ and $\delta T=0.5$ by a factor $\sim 300$.

We have, finally, ascertained the effect of the $\delta$-function broadening. Increasing the Gaussian standard deviation $\Delta E$ from $0.03 \mathrm{eV}$ to $0.06 \mathrm{eV}$ changes the base current $J_{\infty}(0)$ from $1.8 \times 10^{-7} \mu \mathrm{A}$ to $10^{-5} \mu \mathrm{A}$ for $k T_{e}=0.01 \mathrm{eV}$, and from $-0.0062 \mu \mathrm{A}$ to $-0.025 \mu \mathrm{A}$ for $k T_{e}=0.1 \mathrm{eV}$. However, once we calculate the net current $J_{\infty}(\delta T)-J_{\infty}(0)$, that does not change by more than $10 \%$ in the cases considered in Figs. 3 and 4 .

\section{SUMMARY}

We have reexamined the weak-coupling limit of the HP method for electronic open boundaries, in the time domain, and have reduced it to a set of rate equations for electron injection and extraction from the system. These equations are readily combined with rate equations for electron-phonon transitions in the system, to produce a hybrid method involving both. As noted earlier, this is the simplest extension of the HP formalism to time-dependent inelastic transport. 
It has been illustrated above by the calibration of a notional thermometer that registers a temperature difference between system vibrations and the electron baths through the flow of electron current, even at zero bias. We expect, further, the method to be applicable to the calculation of photocurrents [10]. Equation (14) enables the direct calculation of energy transport between the electron and phonon subsystems. It is hoped that this approach will be of interest to the field of nonequilibrium quantum thermodynamics [5].

\section{ACKNOWLEDGMENTS}

We thank Mauro Paternostro and Mads Brandbyge for helpful discussions. We are obliged to Mauro, further, for a critical reading of the manuscript and valuable suggestions. T.N.T. is grateful for support by a research grant from Science Foundation Ireland (SFI) and the Department for the Economy Northern Ireland under the SFI-DfE Investigators Programme Partnership, Grant Number 15/IA/3160.
[1] A. P. Horsfield, M. Boleininger, R. D’Agosta, V. Iyer, A. Thong, T. N. Todorov, and C. White, Phys. Rev. B 94, 075118 (2016).

[2] M. G. Zauchner, A. P. Horsfield, and T. N. Todorov, Phys. Rev. B 97, 045116 (2018).

[3] E. J. McEniry, D. R. Bowler, D. Dundas, A. P. Horsfield, C. G. Sánchez, and T. N. Todorov, J. Phys.: Condens. Matter 19, 196201 (2007).

[4] V. Rizzi, T. N. Todorov, J. J. Kohanoff, and A. A. Correa, Phys. Rev. B 93, 024306 (2016).

[5] G. Benenti, G. Casati, K. Saito, and R. S. Whitney, Phys. Rep. 694, 1 (2017).
[6] J.-T. Lü, J.-S. Wang, P. Hedegård, and M. Brandbyge, Phys. Rev. B 93, 205404 (2016).

[7] A. P. Horsfield, D. R. Bowler, A. J. Fisher, T. N. Todorov, and C. G. Sánchez, J. Phys.: Condens. Matter 17, 4793 (2005).

[8] S. Pigeon, L. Fusco, G. De Chiara, and M. Paternostro, Quantum Sci. Technol. 2, 025006 (2017).

[9] J.-H. Jiang, O. Entin-Wohlman, and Y. Imry, Phys. Rev. B 85, 075412 (2012).

[10] M. Palsgaard, T. Markussen, T. Gunst, M. Brandbyge, and K. Stokbro, Phys. Rev. Applied 10, 014026 (2018). 